\title{
Coupling of a High-Resolution Ambient Pressure Drift Tube Ion Mobility Spectrometer to a Commercial Time-of-Flight Mass Spectrometer
}

\author{
Maria Allers*, Laila Timoumi, Ansgar T. Kirk, Florian Schlottmann, Stefan Zimmermann \\ Leibniz Universität Hannover, Institute of Electrical Engineering and Measurement Technology, Department of \\ Sensors and Measurement Technology, Appelstr. 9A, 30167 Hannover, Germany \\ *Corresponding author: allers@geml.uni-hannover.de
}

\begin{abstract}
Ion mobility spectrometry provides information about molecular structures of ions. Hence, high resolving power allows separation of isomers which is of major interest in several applications. In this work, we couple our high-resolution ion mobility spectrometer (IMS) with a resolving power of $R_{p}=100$ to a time-of-flight mass spectrometer (TOF-MS). Besides the benefit of an increased resolving power such an IMS-MS also helps analyzing and understanding the ionization processes in IMS. Usually, the coupling between IMS and TOF-MS is realized by synchronizing data acquisition of the IMS and MS resulting in two-dimensional data containing ion mobility and mass spectra. However, due to peak widths of less than $100 \mu \mathrm{s}$ in our high-resolution IMS, this technique is not practicable due to significant peak broadening during the ion transfer into the MS and an insufficient data acquisition rate of the MS. Thus, a novel but simple interface between the IMS and MS has been designed which minimizes ion losses, allows recording of ion mobility at full IMS resolving power and enables a shuttered transmission of ions into the MS. The interface is realized by replacing the Faraday plate used in IMS by a Faraday grid that is shielded by two additional aperture grids. For demonstration, positive product ions of benzene, toluene and $m$-xylene in air are investigated. The IMS is equipped with a radioactive ${ }^{3} \mathrm{H}$ source. Besides the well-known product ions $\mathrm{M}^{+}$and $\mathrm{M} \cdot \mathrm{NO}^{+}$, a dimer ion is also observed for benzene and toluene, consisting of two molecules and three further hydrogen atoms.
\end{abstract}

\section{Introduction}

Ion mobility spectrometry (IMS) is a well-known technique for fast online trace gas detection $[1,2]$. Detection limits in the low $\mathrm{ppb}_{\vee}$ (parts per billion) and even $\mathrm{ppt}_{\mathrm{V}}$ (parts per trillion) range combined with fast response times make this technique very interesting for a wide range of applications [3-5]. IMS separates ions based on their mobility in an inert drift gas in the presence of an electric field. In the last years, we developed very compact high-resolution drift tube IMS with short drift lengths between 40 and $150 \mathrm{~mm}[6,7]$ and different ion sources such as radioactive ${ }^{3} \mathrm{H}[8]$ and non-radioactive electron sources [9], corona [7], X-ray [10], ultra violet (UV) [11] and electrospray ionization (ESI) sources [12] reaching a resolving power up to 250 [6]. High-resolution IMS allow the investigation of ions with respect to size, shape and charge gaining insights into molecular structures. In particular, with high IMS resolving power isomers, isobars and conformers can be separated which is of major interest in several applications, such as pharmaceutical, food, fragrance and flavor research, where coupling IMS with mass spectrometry provides a new dimension in the analysis of complex mixtures. In this work, we couple our compact high-resolution ambient pressure IMS to a commercial time-offlight mass spectrometer (Bruker micrOTOF II). Besides the benefit of an increased resolving power our IMS also supports a wide range of interchangeable ion sources, which facilitates the investigation of a wide range of chemical compounds. Thus, this IMS-MS helps analyzing and understanding the ionization processes and eventually identifying the ion species formed in IMS at ambient pressure. 
Over the last 40-50 years, numerous IMS-MS devices have been developed. Different combinations of IMS and MS instruments have been realized, including quadrupole mass spectrometers, ion trap mass spectrometers, Fourier transform mass spectrometers, magnetic sector mass spectrometers, and time-of-flight mass spectrometers as well as High-Field Asymmetric Waveform Ion Mobility Spectrometer (FAIMS), Traveling Wave IMS, Trapped IMS and drift tube IMS with different ion sources operated from low to ambient pressure. Since the original development, a number of commercially available instruments have been developed including those by TOFWERK (IMSTOF), Excellims (RA4100 HPIMS-MS), Agilent (Ion Mobility Q-TOF), Waters Corporation (Synapt-G2) and Bruker (timsTOF), democratizing the field. In particular, the timsTOF has shown high mobility resolving powers when applied to large, multiple-charged ions, reaching resolving powers of up to $R_{p}=250$ [13]. However, for smaller molecules, the drift tube technique reaches better performance. Thus, the aim of this work is to hyphenate our in-house developed drift tube IMS to the commercial Bruker micrOTOF I/ instrument to enable a high-resolution analysis of a wide range of chemical compounds, as our IMS is designed to operate with a variety of interchangeable ion sources. It is also important to note, that our IMS operates at ambient pressure, while most IMS in IMS-MS operate at much low pressure which leads to an increasing effect of $\mathrm{m} / \mathrm{z}$ on ion separation and less separation by collision cross section.

Considering drift tube IMS-TOF-MS couplings in the literature, it can be distinguished between nested and selective techniques [14]. Clemmer and coworkers developed the first fully nested drift tube IMSTOF-MS technique [15]. After being separated in a drift tube, all ions are injected into the mass spectrometer. Since the time frame of an ion mobility spectrum is typically in the millisecond range and the flight time in the mass spectrometer is typically in the microseconds range, for every peak in the ion mobility spectrum several mass spectra can be obtained. This results in a nested twodimensional spectrum containing ion mobility spectra and mass spectra $[16,17]$. The bin size in the drift time is the periodicity of the TOF pulser, within this bin size no differences in drift times are distinguishable. Furthermore, the transfer of ions into the flight tube of the MS leads to peak broadening. Thus, compared to a continuous detector at the end of the IMS drift tube, there is a small loss in IMS resolving power. In common devices, the loss of resolving power is negligible, since the periodicity of the TOF-MS pulser is significantly lower than the IMS peak widths. However, in our compact high-resolution IMS, the peak width is less than $100 \mu \mathrm{s}$ for a resolving power of $R_{p}=100$ [10] or even less than $30 \mu \mathrm{s}$ for a resolving power of $R_{p}=250$ [6]. Thus, peak broadening in the transfer region of the MS becomes more significant decreasing the effective IMS resolving power and the maximum repetition rate of e.g. $20 \mathrm{kHz}$ of the Bruker micrOTOF $/ /$ is no longer sufficient to resolve the ion mobility spectrum.

Nonetheless, in addition to nested IMS-MS techniques, there are selective techniques for transmitting ions of only a selected mobility and then analyzing the resulting mass spectrum. This technique of coupling implies no requirements on the relative analysis times of the coupled devices and is therefore more appropriate for the coupling of our high-resolution IMS to the Bruker micrOTOF II. Early drift tube IMS-MS devices are based on this selective technique using a dual gate ion mobility setup to determine the mobility of ions by only gating out those ions that traversed the drift tube in a predetermined time, and then scanning the delay between the entrance and exit gates $[18,19]$. More recently, Hill and coworkers developed high-resolution IMS-MS devices using a drift tube IMS with two Bradbury-Nielsen gates situated at the entrance and the exit of the drift tube $[20,21]$, which has been applied later in several devices [22-24]. This dual gate technique requires scanning the pulses at the second gate to obtain a full IMS spectrum. Thus, the bin size in the drift time is determined by the minimal gate opening time of the second gate. However, the minimal pulse width of Bradbury-Nielsen shutters of $50 \mu \mathrm{s}$ [25] is insufficient to resolve the ion mobility spectrum of our compact high-resolution IMS. Hence, the full resolving power of our IMS is only maintained when the IMS spectrum is measured directly at the end of the drift tube, as shown in [24] or [26]. 
In this work, a simple IMS-MS interface is presented, minimizing ion losses, allowing the direct measurement of the ion mobility spectra and enabling a shuttered transmission of ions into the MS to investigate single ion peaks and defined time domains of the ion mobility spectrum. Using this interface, it is possible to couple our high-resolution IMS to a Bruker micrOTOF II. Besides maintaining full IMS resolving power important for many applications this IMS-MS allows investigating the ion chemistry employing different ion sources operated at ambient pressure. In this work, positive ions formed by a radioactive ${ }^{3} \mathrm{H}$ source are investigated. As proof of concept study, measurements of benzene, toluene and xylene are presented.

\section{Experimental setup}

\section{Time-of-flight mass spectrometer}

The Bruker micrOTOF II is an Atmospheric Pressure lonization (API) TOF-MS with an ion flight tube containing an orthogonal acceleration stage, a reflector and a microchannel plate detector. Ions from the API source pass through a glass inlet capillary before they enter the first vacuum stage. Using skimmers, RF hexapoles and multi-lens systems, ions are transported through further vacuum stages to the finale pressure stage of the ion flight tube [27]. The transfer parameters are summarized in the supplementary material A. By inversion of the applied voltages, the Bruker micrOTOF // is capable of measuring positive and negative ions. In general, the Bruker micrOTOF I/ is able to measure masses up to $20,000 \mathrm{~m} / \mathrm{z}$. Since this work focusses on the investigation of compounds with low $\mathrm{m} / \mathrm{z}$, the mass range is optimized for masses from 30 to $200 \mathrm{~m} / \mathrm{z}$.

For IMS-MS coupling, the API source is replaced by our high-resolution ambient pressure IMS. Thus, the IMS is directly coupled to the inlet capillary of the MS.

\section{Ion mobility spectrometer}

A detailed description of the compact high-resolution drift tube IMS used in this work is given elsewhere $[6,8]$. It is possible to adapt different ion sources such as radioactive ${ }^{3} \mathrm{H}$ and non-radioactive electron sources, corona as well as X-ray, ultra violet (UV) and electrospray ionization (ESI) sources to the drift tube. In this paper, the drift tube is combined with a radioactive ${ }^{3} \mathrm{H}$ source. In Figure $1 \mathrm{a}$, a schematic of the IMS is shown, and its operating parameters are summarized in Table 1. The basic layout is typical for drift tube designs, consisting of an ionization region, a drift region and a shielded Faraday plate as ion detector. Reactant ion generation is initiated by electron emission from the radioactive ${ }^{3} \mathrm{H}$ source. Ions are injected into the drift region using a field switching shutter as described in [28]. A resistive voltage divider is used for applying the drift field. The whole instrument is sealed inside a surrounding PEEK tube. Dry $\left(<1 \mathrm{ppm}_{\mathrm{v}} \mathrm{H}_{2} \mathrm{O}\right)$, purified air is used as drift gas. The sample gas is directly introduced into the ionization region, while the drift gas enters the drift tube at the detector. The drift region has a length of just $72.5 \mathrm{~mm}$, the IMS resolving power is about $R_{P}=100$. The IMS can be operated in positive and negative ion mode by inversion of the applied voltages.

Table 1: Operating parameters of the IMS

\begin{tabular}{|l|l|}
\hline Parameter & Value \\
\hline Pressure & $1000 \mathrm{mbar}$ \\
\hline Temperature & $30^{\circ} \mathrm{C}$ \\
\hline Drift gas flow & $180 \mathrm{ml} / \mathrm{min}$ \\
\hline Sample gas flow & $20 \mathrm{ml} / \mathrm{min}$ \\
\hline Repetition rate & $44.4 \mathrm{~Hz}$ \\
\hline Drift voltage & $4878 \mathrm{~V}$ \\
\hline Injection voltage & $500 \mathrm{~V}$ \\
\hline Injection time & $150 \mu \mathrm{s}$ \\
\hline
\end{tabular}


a) IMS

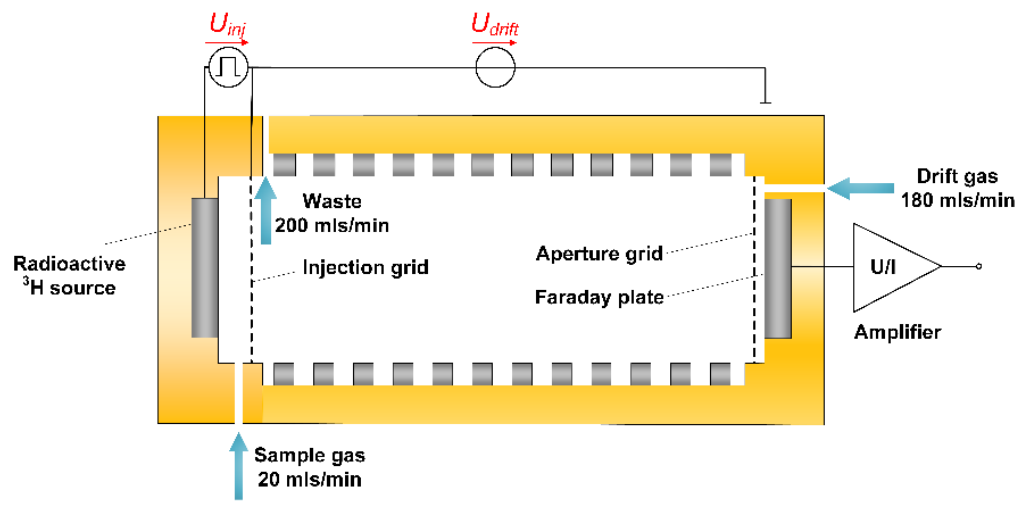

b) IMS coupled to Bruker micrOTOF // mass spectrometer

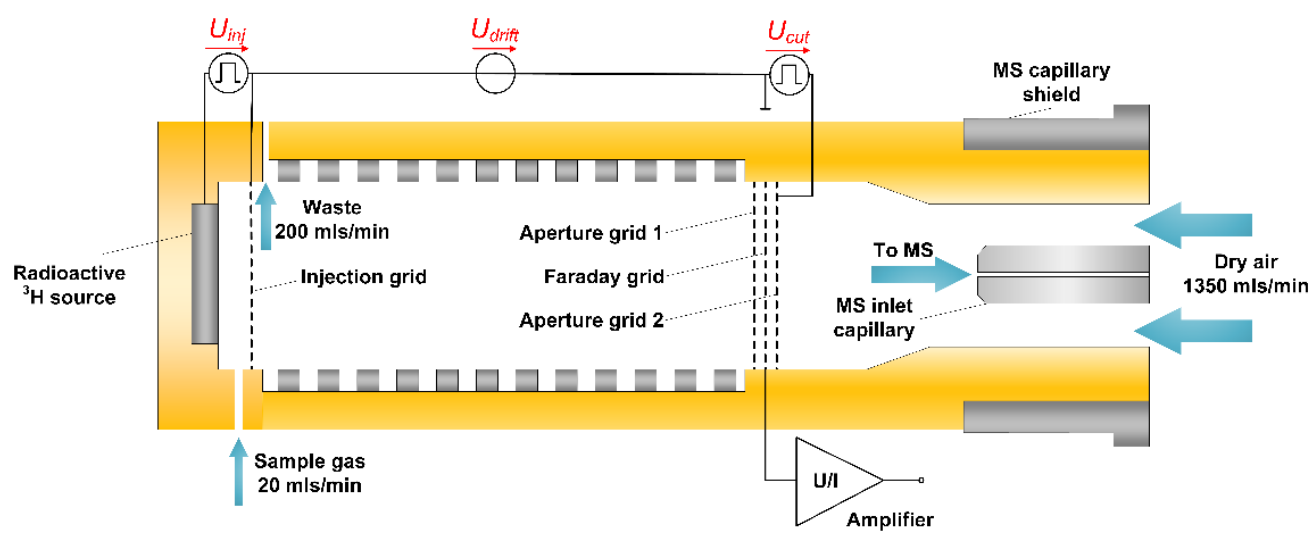

Figure 1: a) Schematic of the high-resolution drift tube IMS (top). b) Schematic of the IMS coupled to the MS inlet capillary (bottom).

\section{Interface}

For simple coupling our IMS to the Bruker micrOTOF // without further modifying the MS, only the Faraday detector of the IMS has to be modified. The novel interface is realized by replacing the Faraday plate of the IMS by a Faraday grid that is shielded by two further aperture grids, as shown in Figure 1b. By directly inserting the interface into the capillary shield of the MS, the IMS can be easily adapted to the MS inlet capillary. The optical transmittance of each grids is $80 \%$. The potentials of the second aperture grid, the MS inlet capillary and the MS capillary shield can be adjusted to control the ratio of ions that discharges at the Faraday grid to the ions transported by electrical fields towards the MS inlet capillary. The inlet gas flow of dry $\left(<1 \mathrm{ppm}_{\vee} \mathrm{H}_{2} \mathrm{O}\right)$, purified air is set to $1350 \mathrm{mls} / \mathrm{min}$ and is split into $180 \mathrm{mls} / \mathrm{min}$ IMS drift gas and $1170 \mathrm{mls} / \mathrm{min}$ MS inlet flow. This high MS inlet flow combined with the small inner diameter of the inlet capillary $(500 \mu \mathrm{m})$ results in very high flow velocities at the MS inlet capillary. Thus, near the capillary, convective ion transport dominates and leads to ion transmission into the MS.

In order to reach maximum ion transmission, ion losses at the second aperture grid and the MS inlet capillary have to be minimized. Therefore, at a given potential of the second aperture grid, an optimal combination of the MS inlet capillary and MS capillary shield potentials has to be found. By increasing the potential of the MS capillary shield, the electrical field lines can be focused onto the MS inlet capillary, as shown in Figure 2. However, increasing the potential of the MS capillary shield also decreases the electric field strength between the MS inlet capillary and the second aperture grid, thus 
leading to increasing ion losses at the second aperture grid. The maximum ion transmission into the MS can be measured for a MS inlet capillary potential of $-4000 \mathrm{~V}$ and a MS capillary shield potential of $-2300 \mathrm{~V}$.

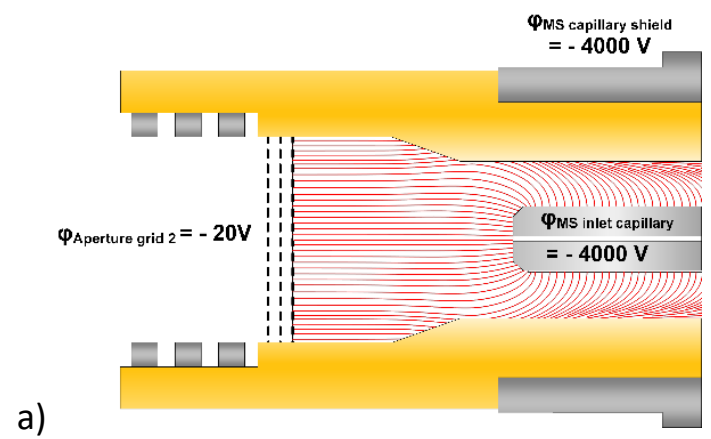

b)

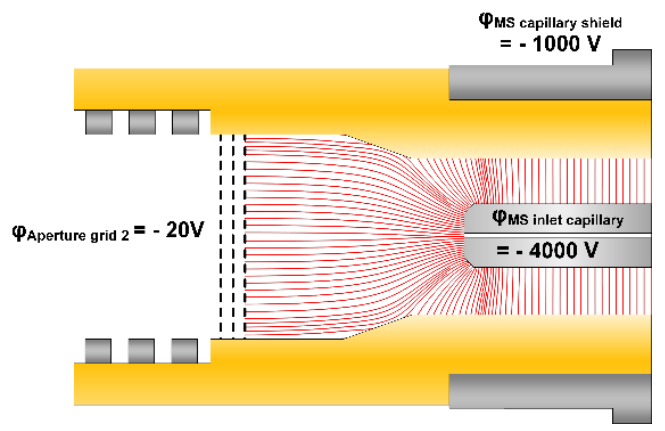

Figure 2: Electrical field lines between the second aperture grid and the MS inlet capillary for a) $-4000 \mathrm{~V}$ and b) -1000 V at the capillary shield.

By varying the potential of the second aperture grid, the transmission of ions into the MS can be controlled. In Figure 3, the measured ion count in the MS in dependence of the potential of the second aperture grid is shown. For a potential of $-20 \mathrm{~V}$, the ion transmission reaches the maximum, for potentials higher than $10 \mathrm{~V}$, the ion transmission is zero. Thus, by switching the electric potential of the second aperture grid $U_{\text {cut }}$ from $10 \mathrm{~V}$ to $-20 \mathrm{~V}$ and back, ion transmission can be turned on and off for a defined time. Hence, it is possible to investigate single ion peaks or defined time domains of the ion mobility spectrum without synchronizing IMS and MS data acquisition. Due to tailored electronics, peak widths in the low microsecond range can be gated.

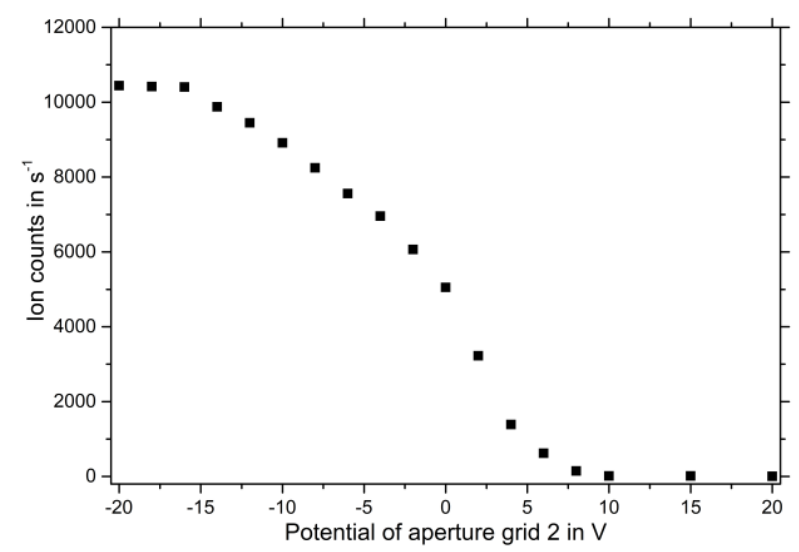

Figure 3: Measured ion count in the MS in dependency of the potential of the second aperture grid (MS inlet capillary potential set to $-4000 \mathrm{~V}, \mathrm{MS}$ capillary shield set to $-2300 \mathrm{~V}$.

However, with decreasing opening times of the ion gate, discrimination of larger ions with lower ion mobility will increase at the ion gate [25]. To overcome this issue, a novel ion gating concept based on a similar three-grid ion gate and a specific waveform with three different states can be used [25].

In summary, this interface allows two operation modes. In the selective mode, the dual gate IMS can be used to transfer single ion peaks or defined time domains of the ion mobility spectrum into the MS to obtain the corresponding mass spectra, as known from [20]. In the IMS mode, the IMS spectrum is measured directly using the Faraday grid. Thus, in contrast to prior dual gate work, in this work, the second gate is not scanned to generate an IMS spectrum, but rather the IMS spectrum is measured directly at the end of the drift tube to maintain full IMS resolving power. 
The ion mobility $K$ is measured experimentally based on the drift time $t_{D}$ it takes an ion to traverse the drift tube of length $L$.

$$
K=\frac{L}{t_{D} \cdot E}
$$

Since the ion mobility $K$ is dependent on both, temperature and pressure, the value calculated in Eq. 1 is often converted to a reduced mobility value $K_{0}$,

$$
K_{0}=K \cdot \frac{p}{1013 m b a r} \cdot \frac{273 K}{T}
$$

where $p$ is the operating pressure in mbar and $T$ is the operating temperature in Kelvin. However, this normalization does not consider variations in ion chemistry due to different conditions in the ionization region, and in particular the variation of moisture content.

\section{Characterization of the IMS-MS system}

\section{Analytical performance}

In Figure 4 , the ion mobility spectrum of dry $\left(<1 p^{2} m_{v} \mathrm{H}_{2} \mathrm{O}\right)$, purified air is shown. The spectrum is measured in IMS mode using the Faraday grid. The peak width at half maximum is $57 \mu$ s leading to a resolving power of about $R_{P}=100$. Thus, the resolving power of our IMS can be maintained using the described interface. Furthermore, the resolving power could be increased to $R_{p}=250$ by replacing the $72.5 \mathrm{~mm}$ drift tube with a compatible $150 \mathrm{~mm}$ drift tube and increasing the drift voltage up to $25 \mathrm{kV}$ as shown in [6]. This requires some modifications of the IMS-MS electronics which will be addressed in the future.

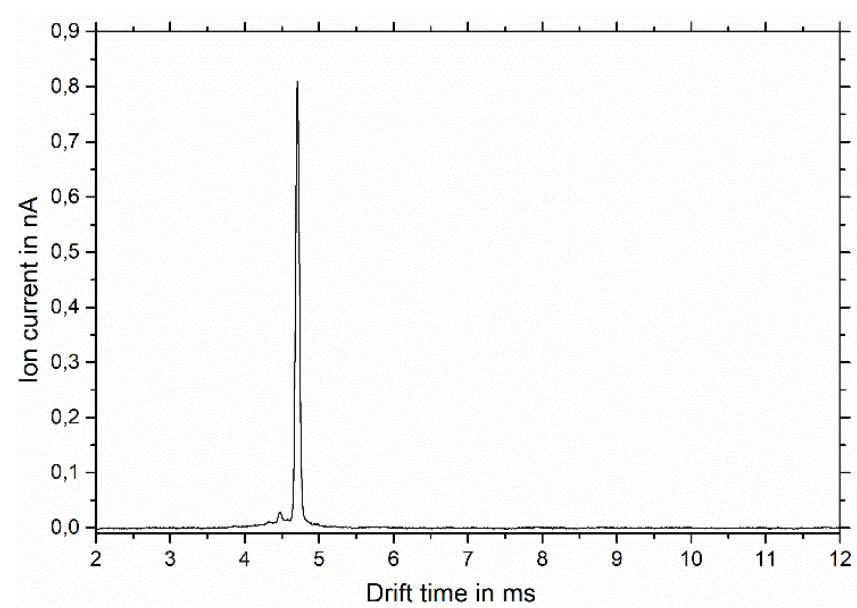

Figure 4: Ion mobility spectrum of dry $\left(<1 p p m_{V} H_{2} O\right)$, purified air. The spectrum is measured in IMS mode using the Faraday grid.

For comparison, the commercial drift tube IMS-TOF-MS devices by Excellims (RA4100 HPIMS-MS) and Agilent (Ion Mobility Q-TOF) reach a resolving power of $R_{p}=60-100$ [29] and $R_{p}=60$ [30] respectively. The device of TOFWERK (IMSTOF) reaches a resolving power of $R_{p}=150$ [31] including a $205 \mathrm{~mm}$ drift tube working at ambient pressure. However, with post-processing, the resolving power exceeds $R_{p}=200[32]$.

Considering ion transmission into the transfer stage of the MS, this simple interface should allow higher ion transmission rates in contrast to systems with a pinhole interface, due to the significantly higher ion transmittance of grids. Comparing this novel interface with a previous pinhole interface we used 
[26], an increase in ion transmission by factor 4 can be observed. However, there are limitations in sensitivity due to the duty cycle of the drift tube IMS. To improve signal intensity and duty cycle, ions could be pulsed into the drift region using a multiplexed gating scheme, as applied in the IMSTOF of TOFWERK [32].

\section{Ion cluster dissociation and ion fragmentation processes in the MS transfer stage}

Being accelerated by electrical fields in the transfer stage of the MS, ions can gain significant kinetic energies between collisions leading to ion cluster dissociation or even ion fragmentation processes [33]. In order to estimate the extent to which ion cluster dissociation or ion fragmentation processes occur in the transfer stage of the Bruker micrOTOF II, the ion peak of the acetone dimer is transferred into the MS and the corresponding mass spectra are recorded for different hexapole RF voltages. In particular, this voltage controls the kinetic energy of the ions. Considering the binding energy of the acetone dimer ion of $1.31 \mathrm{eV} \mathrm{[34]} \mathrm{in} \mathrm{Figure} 5$ demonstrates the impact of the RF amplitude on the mass spectrum. Applying a RF amplitude of $45 \mathrm{~V}$, electrical field driven cluster dissociation processes lead to a predominantly observed bare protonated acetone molecule $(59 \mathrm{u})$, although the peak of the protonated acetone dimer $(117 \mathrm{u})$ is transferred into the MS. Decreasing the RF amplitude, the energy input decreases and thus the measured amount of the bare protonated acetone (59u) decreases. Unfortunately, the ion transmittance into the MS also decreases.
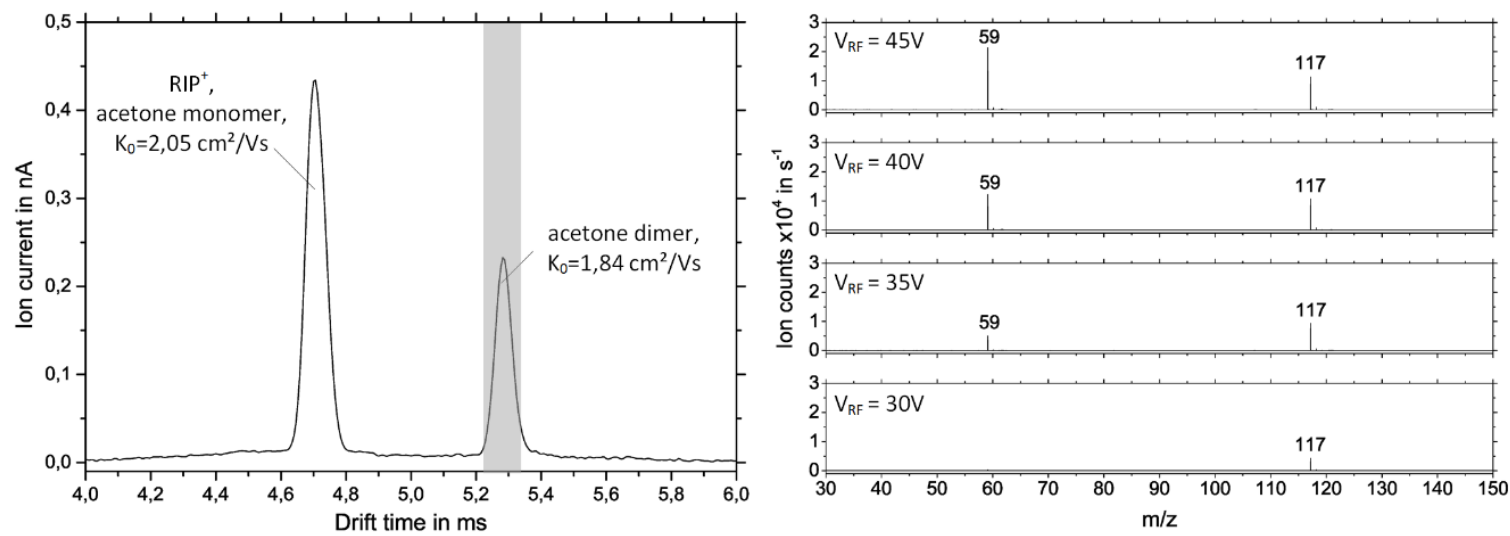

Figure 5: Left: Ion mobility spectrum of $5 p p b_{V}$ acetone in dry $\left(<1 p p m_{V} \mathrm{H}_{2} \mathrm{O}\right)$, purified air. The spectrum is measured in IMS mode using the Faraday grid. The measured reduced mobilities are stated. The acetone monomer cannot be separated from the RIP. Just the acetone dimer is transferred into the MS. Right: Mass spectra of the acetone dimer for different RF voltages of the hexapole.

Due to the partial dissociation of the acetone dimer ion with $1.31 \mathrm{eV}$ binding energy, reduced electrical field strengths up to $100 \mathrm{Td}$ can be assumed in the transfer stage of the Bruker micrOTOF II. Thus, inside the interface, ion fragmentation processes are also possible as known from PTR-MS studies $[35,36]$.

Generally, a high degree of fragmentation is observed for alcohols. Alcohols split off water and break up to non-specific alkane fragments at $\mathrm{m} / \mathrm{z}=43,57$ or 71 [35]. For example, analyzing the 1-octanol monomer with our IMS-MS, this behavior can also be observed as shown in Figure 6 . 

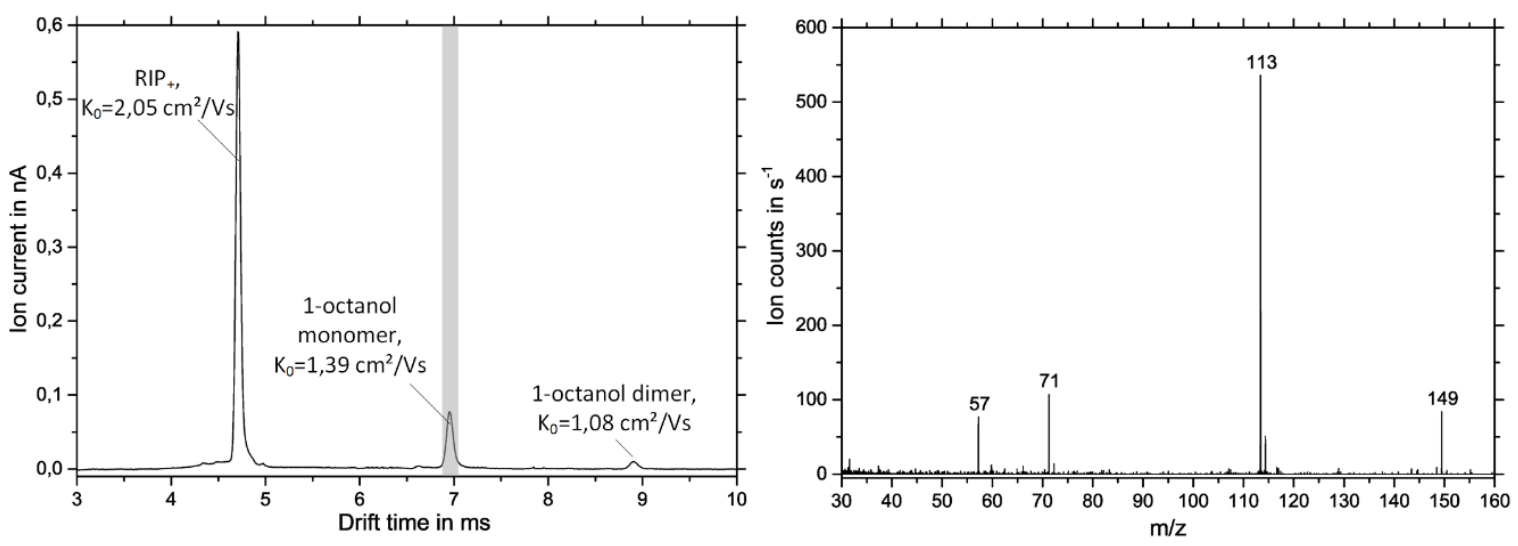

Figure 6: Left: Ion mobility spectrum of $2 p p b_{V}$ 1-octanol in dry $\left(<1 p p m_{V} \mathrm{H}_{2} \mathrm{O}\right)$, purified air. The spectrum is measured in IMS mode using the Faraday grid. The measured reduced mobilities are stated. The 1-octanol monomer is transferred into the MS. Right: Mass spectrum of the 1-octanol monomer.

The molecular mass of 1-octanol is $130 \mathrm{u}$. In the mass spectrum, the most abundant peak is $113 \mathrm{~m} / \mathrm{z}$, which results from the protonated molecule minus water $(130 u-18 u+1 u=113 u)$. Additionally, the protonated molecule plus water is present $(130 u+18 u+1 u=149 u)$. Furthermore, alkane fragments at $\mathrm{m} / \mathrm{z}=57$ and $\mathrm{m} / \mathrm{z}=71$ can be observed.

Additionally, investigating the positive reactant ions and the corresponding mass spectra cluster dissociation processes in the transfer stage of the mass spectrometer can be observed, as shown in Figure 7.
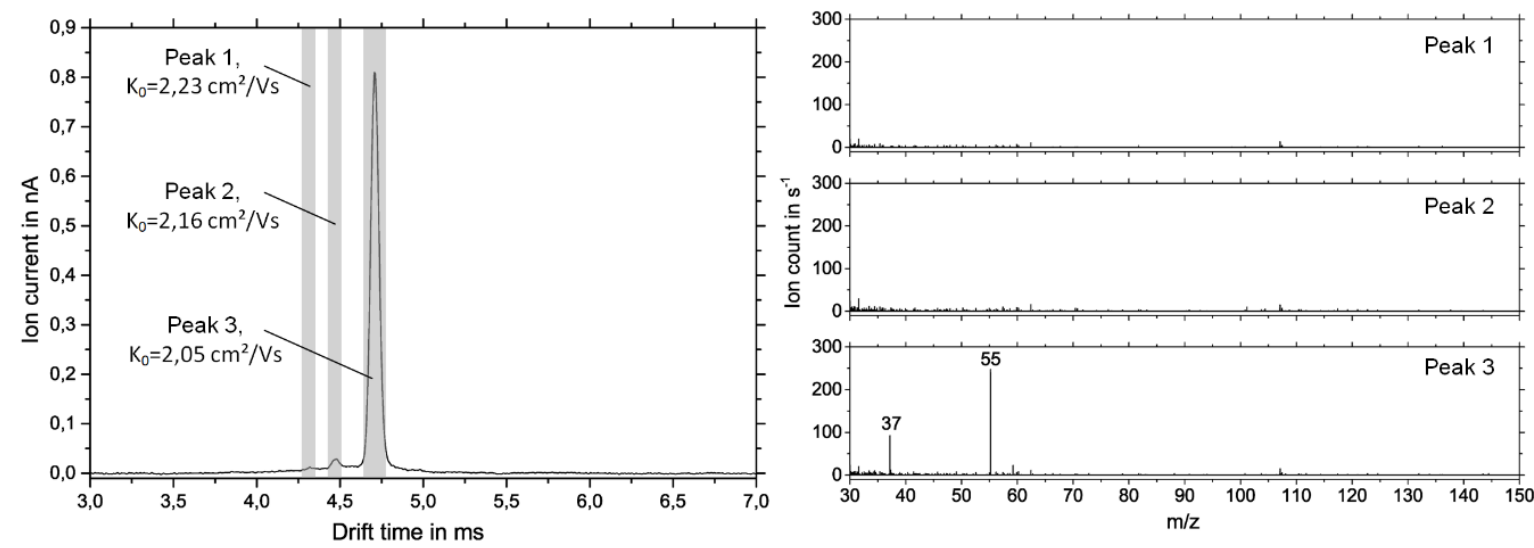

Figure 7: Left: Ion mobility spectrum of dry $\left(<1 \mathrm{ppm}_{V} \mathrm{H}_{2} \mathrm{O}\right)$, purified air. The spectrum is measured in IMS mode using the Faraday grid. The measured reduced mobilities are stated. Right: The corresponding mass spectra.

The ion mobility spectrum of dry $\left(<1 \mathrm{ppm}_{\mathrm{v}} \mathrm{H}_{2} \mathrm{O}\right)$, purified air shows one major peak $\left(\mathrm{K}_{0}=2,05 \mathrm{~cm}^{2} / \mathrm{Vs}\right)$ attributed to the positive reactant ion peak $\left(\mathrm{RIP}^{+}\right)$accompanied by two smaller peaks $\left(\mathrm{K}_{0}=2,16 \mathrm{~cm}^{2} / \mathrm{Vs}\right.$ and $\left.\mathrm{K}_{0}=2,23 \mathrm{~cm}^{2} / \mathrm{Vs}\right)$. It is well known, that the ion chemistry in atmospheric pressure chemical ionization sources is dominated by ion clustering processes, especially those that involve water molecules. In previous work, the three reactant ion peaks have been identified as $\mathrm{NH}_{4}{ }^{+}\left(\mathrm{H}_{2} \mathrm{O}\right)_{n}$, $\mathrm{NO}^{+}\left(\mathrm{H}_{2} \mathrm{O}\right)_{n}$ and $\mathrm{H}^{+}\left(\mathrm{H}_{2} \mathrm{O}\right)_{n}$ [37-39]. However, in the corresponding mass spectra, apart from $\mathrm{H}^{+}\left(\mathrm{H}_{2} \mathrm{O}\right)_{n}$ ions, no other ions are observed.

Kebarle et. al and many other research groups have investigated and quantitatively described the thermodynamic and kinetic properties of the thermalized proton bound water cluster system [37,4042]. Based on this literature and kinetic simulations [43], proton bound water clusters with $n=4$ are expected as major species in a thermally equilibrated system at atmospheric pressure and $<1 \mathrm{ppm}_{V}$ humidity. However, in the corresponding mass spectrum, the highest abundance of water clusters is 
measured for the $\mathrm{H}^{+}\left(\mathrm{H}_{2} \mathrm{O}\right)_{3}$ ions $(55 \mathrm{u})$. Additionally, $\mathrm{H}^{+}\left(\mathrm{H}_{2} \mathrm{O}\right)_{2}$ ions $(37 \mathrm{u})$ are present in the mass spectrum. Thus, it becomes obvious that cluster dissociation processes occur inside the transfer stage and ions gain enough kinetic energy to break the binding between $\mathrm{H}^{+}\left(\mathrm{H}_{2} \mathrm{O}\right)_{3}$ and $\mathrm{H}_{2} \mathrm{O}$ (binding energy of $0.78 \mathrm{eV}$ [41]), the binding between $\mathrm{H}^{+}\left(\mathrm{H}_{2} \mathrm{O}\right)_{2}$ and $\mathrm{H}_{2} \mathrm{O}$ (binding energy of $0.85 \mathrm{eV}$ [41]) and to some extent to break the binding between $\mathrm{H}_{3} \mathrm{O}^{+}$and $\mathrm{H}_{2} \mathrm{O}$ (binding energy of $1.38 \mathrm{eV}$ [41]). However, the single protonated water molecule $\mathrm{H}_{3} \mathrm{O}^{+}(19 \mathrm{u})$ cannot be detected due to the mass discrimination of the MS used in this work. Since the binding energy of $\mathrm{NH}_{4}{ }^{+}$with $\mathrm{H}_{2} \mathrm{O}$ is about $0.75 \mathrm{eV}$ [44] and the binding energy of $\mathrm{NO}^{+}$with $\mathrm{H}_{2} \mathrm{O}$ is about $0.81 \mathrm{eV}$ [45], the bare $\mathrm{NH}_{4}{ }^{+}$and $\mathrm{NO}^{+}$ions are expected to be present after the transfer stage. Again, due to the mass discrimination of small molecules, $\mathrm{NH}_{4}{ }^{+}$and $\mathrm{NO}^{+}$cannot be detected by the MS.

These examples show the occurrence of ion cluster dissociation and ion fragmentation processes inside the transfer stage of the Bruker micrOTOF I/ mass spectrometer. Thus, when analyzing the ion mobility spectra and the corresponding mass spectra, one has to be aware that the mass spectrum might not show the ions present in the IMS. However, IMS-MS data help to better understand the ion formation in ambient pressure IMS.

\section{Results}

As proof-of-concept study, the presented IMS-MS is used to investigate the product ions of benzene, toluene and m-xylene in dry $\left(<1 \mathrm{ppm}_{\mathrm{v}} \mathrm{H}_{2} \mathrm{O}\right)$, purified air. The results are shown in Figure 8 . As known from previous work $[46,47]$, the low ionization energy of aromatic compounds compared to the ionization energy of $\mathrm{NO}^{+}$allows charge transfer reactions leading to single charged $\mathrm{M}^{+}$ions. In addition, adduct ions $\mathrm{M} \cdot \mathrm{NO}^{+}$are formed. Proton transfer or ligand switching reactions with $\mathrm{H}^{+}\left(\mathrm{H}_{2} \mathrm{O}\right)_{n}$ reactant ions and $n>2$ are not possible for benzene or toluene due to their low proton affinities and small dipole moments $[46,48]$. In contrast, for $\mathrm{m}$-xylene, both the single charged $\mathrm{M}^{+}$ions and the protonated $\mathrm{MH}^{+}$ ions can be observed in the ion mobility spectrum.

Furthermore, for high analyte concentrations ( $>1$ ppmv), a dimer peak is present in the ion mobility spectrum of benzene and toluene. In both cases, this peak consists of two molecules and three hydrogen atoms resulting in $\mathrm{m} / \mathrm{z}=159$ for benzene $((2.78 \mathrm{u})+3 \mathrm{u}=159 \mathrm{u})$ and $\mathrm{m} / \mathrm{z}=187$ for toluene $((2.92 u)+3 u=187 u)$. However, such a dimer peak cannot be detected for $m$-xylene even at very high xylene concentrations. Moreover, this dimer seems to be less stable for toluene in comparison to benzene. In previous work, the benzene dimer is observed to consists of two benzene molecules $[49,50]$. So far, to the best of our knowledge, the threefold hydrogenated dimer has not been reported for aromatic hydrocarbons. Thus, the formation process of this dimer ion is unknown and needs to be further investigated. 
a)

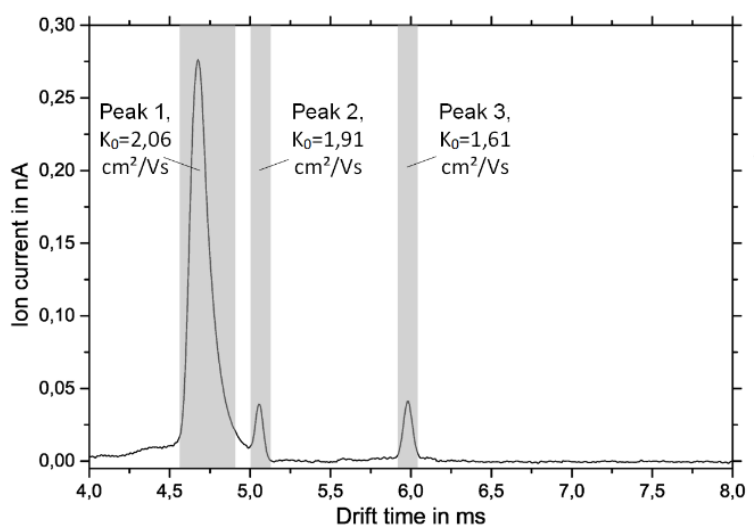

b)

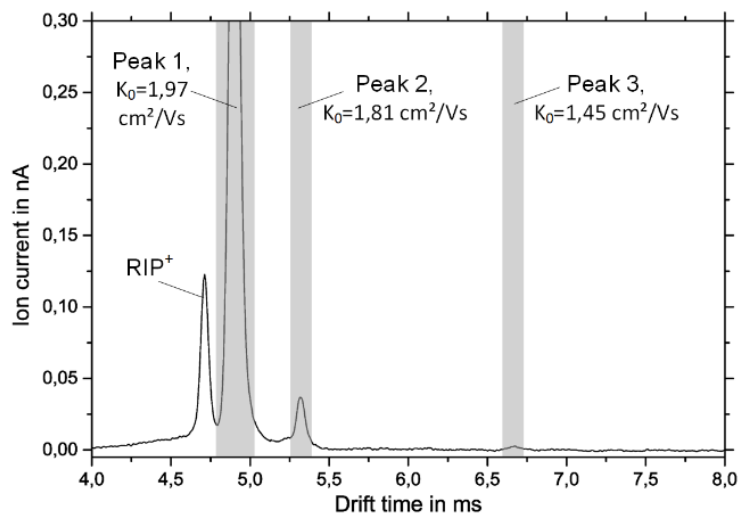

c)

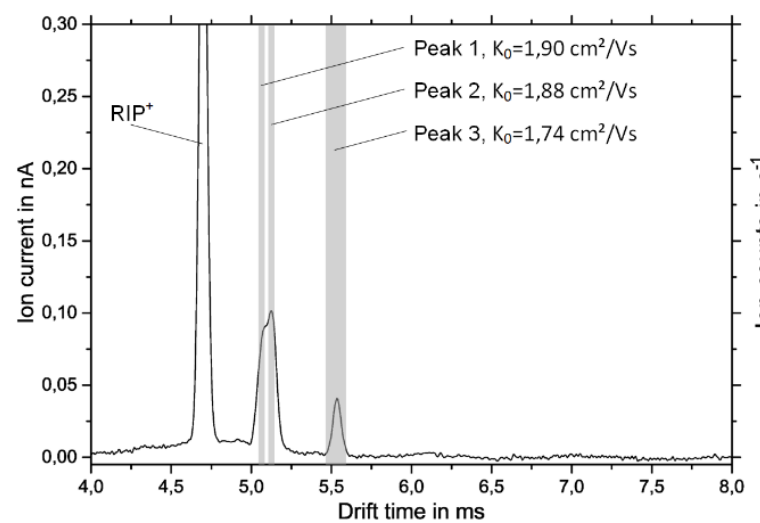

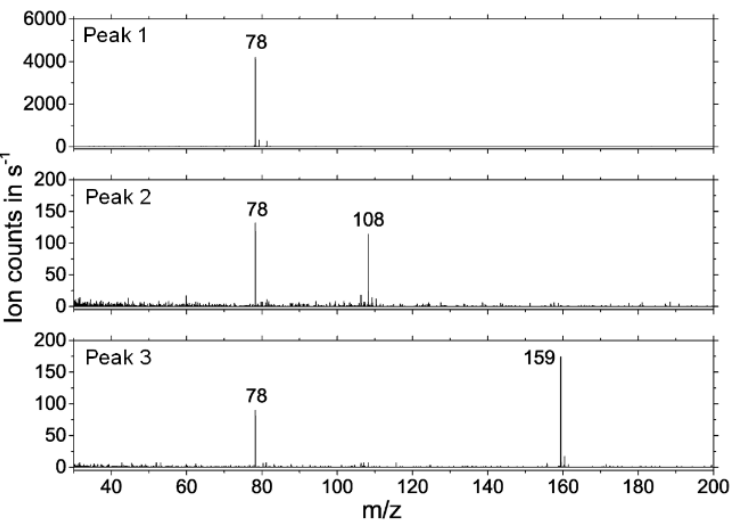
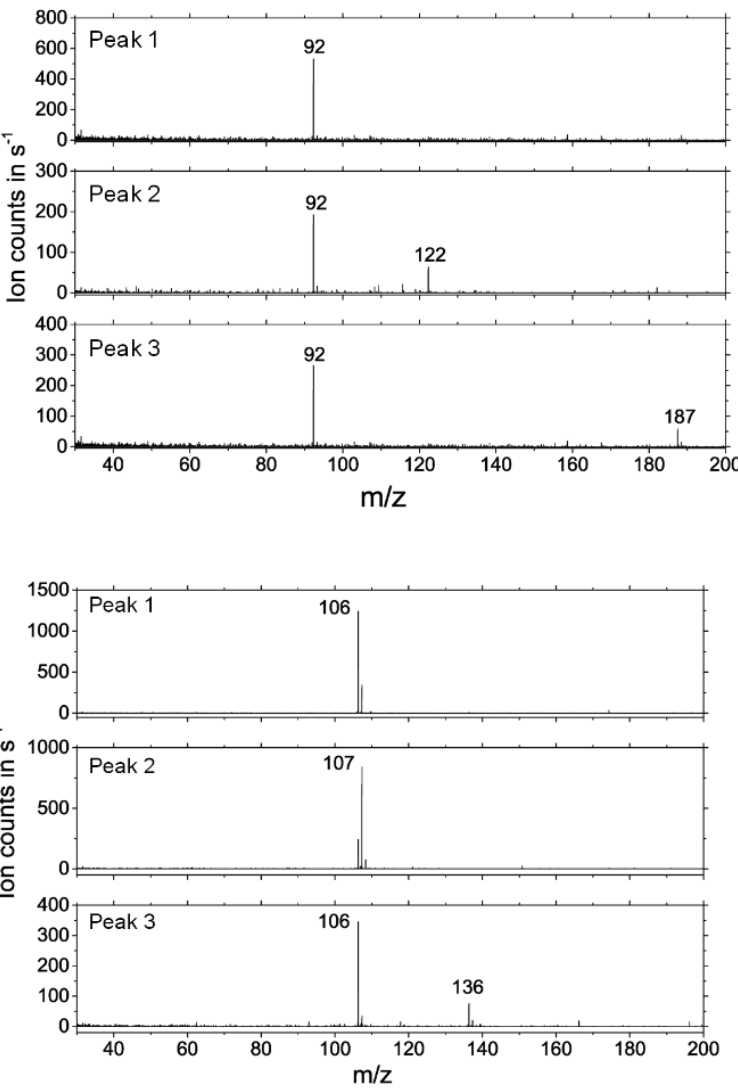

Figure 8: Left: Ion mobility spectrum of a) $7 p p m_{V}$ benzene, b) $9 p p m_{V}$ toluene and c) $2 p p m_{V} m$-xylene in dry (< $\left.1 p p m_{V} \mathrm{H}_{2} \mathrm{O}\right)$, purified air. The spectra are measured in IMS mode using the Faraday grid. The measured reduced mobilities are stated. Right: Corresponding mass spectra.

\section{Conclusion}

In this work, a simple IMS-MS interface to couple our compact high-resolution ambient pressure IMS (drift length of just $72 \mathrm{~mm}$, resolving power of $R_{p}=100$ ) to a Bruker micrOTOF II mass spectrometer is presented. The interface is realized by replacing the Faraday plate of the IMS by a Faraday grid that is shielded by two further aperture grids. Due to the high ion transmittance of the used grids in contrast to pinholes, ion losses are minimized. Furthermore, this interface allows recording the ion mobility spectrum at full resolving power and enables a gated transmission of ions into the MS to investigate single ion peaks or defined time domains of the ion mobility spectrum by simply varying the potential of the second aperture grid. Due to tailored electronics, peak widths in the low microsecond range can be gated. Considering the positive ions formed by a radioactive ${ }^{3} \mathrm{H}$ source, the IMS-MS data show the expected ion cluster dissociation and ion fragmentation processes inside the transfer stage of the 
Bruker micrOTOF // mass spectrometer. Reduced electrical field strengths up to $100 \mathrm{Td}$ can be assumed in the transfer stage. As proof-of-concept study, measurements of benzene, toluene and xylene in dry $\left(<1\right.$ ppm $\left._{v} \mathrm{H}_{2} \mathrm{O}\right)$, purified air are presented. Besides the well-known product ions $\mathrm{M}^{+}$and $\mathrm{M} \cdot \mathrm{NO}^{+}, \mathrm{a}$ dimer ion can be observed for high benzene and toluene concentrations, consisting of two molecules and three hydrogen atoms. Future work aims for investigating the ionization processes at ambient pressure of different ion sources such as radioactive ${ }^{3} \mathrm{H}$ and non-radioactive electron sources, corona, X-ray, ultra violet (UV) and electrospray ionization (ESI) sources.

\section{Acknowledgments}

This work was funded by the Deutsche Forschungsgemeinschaft (DFG, German Research Foundation) - ZI 1288/8-1 


\section{References}

[1] H. Borsdorf, T. Mayer, M. Zarejousheghani, G. A. Eiceman (2011): Recent Developments in Ion Mobility Spectrometry, Applied Spectroscopy Reviews 46, 472-521; doi: 10.1080/05704928.2011.582658.

[2] G.A. Eiceman, Z. Karpas, H.H. Hill: Ion mobility spectrometry. 3rd ed., 3rd ed., CRC Press, Boca Raton, 2013.

[3] J. I. Baumbach (2009): Ion mobility spectrometry coupled with multi-capillary columns for metabolic profiling of human breath, Journal of breath research 3, 34001; doi: 10.1088/1752$7155 / 3 / 3 / 034001$.

[4] I. A. Buryakov (2011): Detection of explosives by ion mobility spectrometry, J Anal Chem 66, 674-694; doi: 10.1134/S1061934811080077.

[5] M. Hernández-Mesa, A. Escourrou, F. Monteau, B. Le Bizec, G. Dervilly-Pinel (2017): Current applications and perspectives of ion mobility spectrometry to answer chemical food safety issues, TrAC Trends in Analytical Chemistry 94, 39-53; doi: 10.1016/j.trac.2017.07.006.

[6] A. T. Kirk, S. Zimmermann (2015): Pushing a compact $15 \mathrm{~cm}$ long ultra-high resolution drift tube ion mobility spectrometer with $R=250$ to $R=425$ using peak deconvolution, Int. J. Ion Mobil. Spec. 18, 17-22; doi: 10.1007/s12127-015-0166-z.

[7] A. Ahrens, S. Zimmermann (Eds.): A5.2 - A miniaturized drift tube ion mobility spectrometer for hand-held devices. AMA Service GmbH, Von-Münchhausen-Str. 49, 31515 Wunstorf, Germany, 2017.

[8] A. T. Kirk, M. Allers, P. Cochems, J. Langejuergen, S. Zimmermann (2013): A compact high resolution ion mobility spectrometer for fast trace gas analysis, Analyst 138, 5200-5207; doi: 10.1039/c3an00231d.

[9] P. Cochems, M. Runge, S. Zimmermann (2014): A current controlled miniaturized nonradioactive electron emitter for atmospheric pressure chemical ionization based on thermionic emission, Sensors and Actuators A: Physical 206, 165-170; doi: 10.1016/j.sna.2013.11.033.

[10] T. Reinecke, A. T. Kirk, A. Heptner, D. Niebuhr, S. Bottger, S. Zimmermann (2016): A compact high-resolution X-ray ion mobility spectrometer, Rev. Sci. Instrum. 87, 53120; doi: 10.1063/1.4950866.

[11] A. T. Kirk, C.-R. Raddatz, S. Zimmermann (2017): Separation of Isotopologues in Ultra-HighResolution Ion Mobility Spectrometry, Anal. Chem. 89, 1509-1515; doi:

10.1021/acs.analchem.6b03300.

[12] T. Reinecke, A. T. Kirk, A. Ahrens, C.-R. Raddatz, C. Thoben, S. Zimmermann (2016): A compact high resolution electrospray ionization ion mobility spectrometer, Talanta 150, 1-6; doi: 10.1016/j.talanta.2015.12.006.

[13] J. A. Silveira, M. E. Ridgeway, M. A. Park (2014): High resolution trapped ion mobility spectrometery of peptides, Anal. Chem. 86, 5624-5627; doi: 10.1021/ac501261h.

[14] M. A. Ewing, M. S. Glover, D. E. Clemmer (2016): Hybrid ion mobility and mass spectrometry as a separation tool, J. Chrom. A 1439, 3-25; doi: 10.1016/j.chroma.2015.10.080. 
[15] C. S. Hoaglund, S. J. Valentine, C. R. Sporleder, J. P. Reilly, D. E. Clemmer (1998): ThreeDimensional Ion Mobility/TOFMS Analysis of Electrosprayed Biomolecules, Anal. Chem. 70, 22362242; doi: 10.1021/ac980059c.

[16] S. C. Henderson, S. J. Valentine, A. E. Counterman, D. E. Clemmer (1999): ESI/Ion Trap/Ion Mobility/Time-of-Flight Mass Spectrometry for Rapid and Sensitive Analysis of Biomolecular Mixtures, Anal. Chem. 71, 291-301; doi: 10.1021/ac9809175.

[17] W. E. Steiner, B. H. Clowers, K. Fuhrer, M. Gonin, L. M. Matz, W. F. Siems, A. J. Schultz, H. H. Hill (2001): Electrospray ionization with ambient pressure ion mobility separation and mass analysis by orthogonal time-of-flight mass spectrometry, Rapid Commun. Mass Spectrom. 15, 2221-2226; doi: $10.1002 / \mathrm{rcm} .495$.

[18] F. W. Karasek, H. H. Hill, S. H. Kim (1976): Plasma chromatography of heroin and cocaine with mass-identified mobility spectra, J Chromatogr 117, 327-336.

[19] E. W. McDaniel, D. W. Martin, W. S. Barnes (1962): Drift Tube-Mass Spectrometer for Studies of Low-Energy Ion-Molecule Reactions, Rev. Sci. Instrum. 33, 2-7; doi: 10.1063/1.1717656.

[20] P. Dwivedi, B. Bendiak, B. H. Clowers, H. H. Hill (2007): Rapid resolution of carbohydrate isomers by electrospray ionization ambient pressure ion mobility spectrometry-time-of-flight mass spectrometry (ESI-APIMS-TOFMS), J Am Soc Mass Spectrom 18, 1163-1175; doi: 10.1016/j.jasms.2007.04.007.

[21] B. H. Clowers, H. H. Hill JR (2005): Mass analysis of mobility-selected ion populations using dual gate, ion mobility, quadrupole ion trap mass spectrometry, Anal. Chem. 77, 5877-5885; doi: 10.1021/ac050700s.

[22] J. D. Keelor, S. Zambrzycki, A. Li, B. H. Clowers, F. M. Fernández (2017): Atmospheric Pressure Drift Tube Ion Mobility-Orbitrap Mass Spectrometry, Analytical chemistry 89, 11301-11309; doi: 10.1021/acs.analchem.7b01866.

[23] P. Dwivedi, A. J. Schultz, H. H. Hill (2010): Metabolic Profiling of Human Blood by High Resolution Ion Mobility Mass Spectrometry (IM-MS), Int. J. Mass Spectrom. 298, 78-90; doi: 10.1016/j.ijms.2010.02.007.

[24] X. Tang, J. E. Bruce, H. H. Hill (2007): Design and performance of an atmospheric pressure ion mobility Fourier transform ion cyclotron resonance mass spectrometer, Rapid Communications in Mass Spectrometry 21, 1115-1122; doi: 10.1002/rcm.2928.

[25] A. T. Kirk, D. Grube, T. Kobelt, C. Wendt, S. Zimmermann (2018): A High Resolution High Kinetic Energy Ion Mobility Spectrometer Based on a Low-Discrimination Tri-State Ion Shutter, Anal. Chem. 90, 5603-5611; doi: 10.1021/acs.analchem.7b04586.

[26] A. Heptner, T. Reinecke, J. Langejuergen, S. Zimmermann (2014): A gated atmospheric pressure drift tube ion mobility spectrometer-time-of-flight mass spectrometer, J. Chrom. A 1356, 241-248; doi: 10.1016/j.chroma.2014.06.059.

[27] Bruker Daltonics: micrOTOF II User Manual, 2008.

[28] A. T. Kirk, S. Zimmermann (2014): Bradbury-Nielsen vs. Field switching shutters for high resolution drift tube ion mobility spectrometers, Int. J. Ion Mobil. Spec. 17, 131-137; doi: 10.1007/s12127-014-0153-9.

[29] Excellims: Product information for RA4100. 
[30] Agilent Technologies: Agilent 6560 Ion Mobility Q-TOF Specifications: Data Sheet.

[31] R. Knochenmuss, S. Graf, K. Fuhrer, M. Gonin: Multiplexed High Pressure Ion MobilityTOFMS: High Resolution, Sensitivity and Structural Information in One Package, in: Proceedings of the 61st ASMS Conference on Mass Spectrometry, 2013.

[32] J. E. Krechmer, M. Groessl, X. Zhang, H. Junninen, P. Massoli, A. T. Lambe, J. R. Kimmel, M. J. Cubison, S. Graf, Y.-H. Lin, S. H. Budisulistiorini, H. Zhang, J. D. Surratt, R. Knochenmuss, J. T. Jayne, D. R. Worsnop, J.-L. Jimenez, M. R. Canagaratna (2016): Ion mobility spectrometry-mass spectrometry (IMS-MS) for on- and offline analysis of atmospheric gas and aerosol species, Atmos. Meas. Tech. 9, 3245-3262; doi: 10.5194/amt-9-3245-2016.

[33] C. Warneke, van der Veen, C, S. Luxembourg, de Gouw, J. A, A. Kok (2001): Measurements of benzene and toluene in ambient air using proton-transfer-reaction mass spectrometry: calibration, humidity dependence, and field intercomparison, Int. J. Mass Spectrom. 207, 167-182.

[34] Y. K. Lau, P. P. S. Saluja, P. Kebarle (1980): The proton in dimethyl sulfoxide and acetone. Results from gas-phase ion equilibriums involving (Me2SO) $\mathrm{nH}+$ and $(\mathrm{Me} 2 \mathrm{CO}) \mathrm{nH}+$, J. Am. Chem. Soc. 102, 7429-7433; doi: 10.1021/ja00545a004.

[35] M. Gueneron, M. H. Erickson, G. S. VanderSchelden, B. T. Jobson (2015): PTR-MS fragmentation patterns of gasoline hydrocarbons, Int. J. Mass Spectrom. 379, 97-109; doi: 10.1016/j.ijms.2015.01.001.

[36] K. Buhr, S. van Ruth, C. Delahunty (2002): Analysis of volatile flavour compounds by Proton Transfer Reaction-Mass Spectrometry: fragmentation patterns and discrimination between isobaric and isomeric compounds, Int. J. Mass Spectrom. 221, 1-7; doi: 10.1016/S1387-3806(02)00896-5.

[37] Eiceman, G.A., Nazarov, E.G., Rodriguez, J.E., and Bergloff, J.F (1998): Positive Reactant lon Chemistry for Analytical, High Temperature Ion Mobility Spectrometry IMS Effects of Electric Field of the Drift Tube and Moisture, Temperature, and Flow of the Drift Gas, Int. J. Ion Mobil. Spec.

[38] D. I. Carroll, I. Dzidic, R. N. Stillwell, E. C. Horning (1975): Identification of positive reactant ions observed for nitrogen carrier gas in plasma chromatograph mobility studies 47, 1956-1959; doi: 10.1021/ac60362a029.

[39] S. H. Kim, K. R. Betty, F. W. Karasek (1978): Mobility behavior and composition of hydrated positive reactant ions in plasma chromatography with nitrogen carrier gas 50, 2006-2012; doi: 10.1021/ac50036a018.

[40] M. Pavlik, J. D. Skalny (1997): Generation of [H3O]+.(H2O)n clusters by positive corona discharge in air, Rapid Commun. Mass Spectrom. 11, 1757-1766; doi: 10.1002/(SICI)10970231(19971030)11:16<1757:AID-RCM16>3.0.CO;2-8.

[41] P. Kebarle, S. K. Searles, A. Zolla, J. Scarborough, M. Arshadi (1967): Solvation of the hydrogen ion by water molecules in the gas phase. Heats and entropies of solvation of individual reactions. $\mathrm{H}+(\mathrm{H} 2 \mathrm{O}) \mathrm{n}-1+\mathrm{H} 2 \mathrm{O}$.fwdarw. $\mathrm{H}+(\mathrm{H} 2 \mathrm{O})$ n, J. Am. Chem. Soc. 89, 6393-6399; doi: 10.1021/ja01001a001.

[42] Y. K. Lau, S. Ikuta, P. Kebarle (1982): Thermodynamics and kinetics of the gas-phase reactions $\mathrm{H} 3 \mathrm{O}+(\mathrm{H} 2 \mathrm{O}) \mathrm{n}-1+$ water $=\mathrm{H} 3 \mathrm{O}+(\mathrm{H} 2 \mathrm{O}) \mathrm{n}, \mathrm{J}$. Am. Chem. Soc. 104, 1462-1469; doi: 10.1021/ja00370a002.

[43] S. Klee, V. Derpmann, W. Wissdorf, S. Klopotowski, H. Kersten, K. J. Brockmann, T. Benter, S. Albrecht, A. P. Bruins, F. Dousty, T. J. Kauppila, R. Kostiainen, R. O’Brien, D. B. Robb, J. A. Syage 
(2014): Are clusters important in understanding the mechanisms in atmospheric pressure ionization? Part 1: Reagent ion generation and chemical control of ion populations, J. Am. Soc. Mass Spectrom. 25, 1310-1321; doi: 10.1007/s13361-014-0891-2.

[44] J. D. Payzant, A. J. Cunningham, P. Kebarle (1973): Gas Phase Solvation of the Ammonium Ion by NH 3 and $\mathrm{H} 2 \mathrm{O}$ and Stabilities of Mixed Clusters NH 4+ (NH 3 ) n (H 2 O) w, Can. J. Chem. 51, 3242-3249; doi: 10.1139/v73-485.

[45] M. A. French, L. P. Hills, P. Kebarle (1973): Kinetics and Temperature Dependence of the Hydration of NO + in the Gas Phase, Can. J. Chem. 51, 456-461.

[46] J. Langejuergen, M. Allers, J. Oermann, A. T. Kirk, S. Zimmermann (2014): Quantitative detection of benzene in toluene- and xylene-rich atmospheres using high-kinetic-energy ion mobility spectrometry (IMS), Anal. Chem. 86, 11841-11846; doi: 10.1021/ac5034243.

[47] P. Španěl, D. Smith (1998): Selected ion flow tube studies of the reactions of $\mathrm{H} 3 \mathrm{O}+, \mathrm{NO}+$, and $\mathrm{O} 2+$ with several aromatic and aliphatic hydrocarbons, Int. J. Mass Spectrom. 181, 1-10; doi: 10.1016/S1387-3806(98)14114-3.

[48] A. J. Midey, S. Williams, S. T. Arnold, A. A. Viggiano (2002): Reactions of H3O+(H2O)0,1 with Alkylbenzenes from 298 to 1200 K, J. Phys. Chem. A 106, 11726-11738.

[49] K. Hiraoka, S. Fujimaki, K. Aruga, S. Yamabe (1991): Stability and structure of benzene dimer cation (C 6 H 6 ) +2 in the gas phase, J. Chem. Phys. 95, 8413-8418; doi: 10.1063/1.461270.

[50] K. C. Janda, J. C. Hemminger, J. S. Winn, S. E. Novick, S. J. Harris, W. Klemperer (1975): Benzene dimer: A polar molecule, J. Chem. Phys. 63, 1419-1421; doi: 10.1063/1.431502. 